\title{
Domesticity in projective spaces
}

\author{
Beukje Temmermans* Joseph A. Thas \\ Hendrik Van Maldeghem
}

full screen

close

quit

\begin{abstract}
Let $J$ be a set of types of subspaces of a projective space. Then a collineation or a duality is called $J$-domestic if it maps no flag of type $J$ to an opposite one. In this paper, we characterize symplectic polarities as the only dualities of projective spaces that map no chamber to an opposite one. This implies a complete characterization of all $J$-domestic dualities of an arbitrary projective space for all type subsets $J$. We also completely characterize and classify $J$-domestic collineations of projective spaces for all possible $J$.
\end{abstract}

Keywords: symplectic polarity, displacement, projective spaces

MSC 2000: 51A10

\section{Introduction}

Abramenko and Brown [1] show that every automorphism of an irreducible non-spherical building has infinite displacement. Their method also gives information about the spherical case. For instance, in the rank 2 case, every automorphism maps some chamber to a chamber at codistance one, and if the diameter of the incidence graph is even (odd), then any duality (collineation) maps some chamber to an opposite one. For projective planes, this shows that collineations behave normally, where 'normal' means that at least one chamber is mapped onto an opposite one. However, it is easily seen that also dualities of projective planes behave normally. Counterexamples to this normal behaviour

\footnotetext{
*The first author is supported by the Fund for Scientific Research - Flanders (FWO - Vlaanderen), Research Project G.0086.06
} 



\section{Domestic dualities}

In this section we will prove the following theorem.

Theorem 3.1. Every domestic duality of a projective space is a symplectic polarity. In particular no even dimensional projective space admits domestic dualities.

It is clear that in any one-dimensional projective space, the only domestic collineation is the identity, and for any domestic duality, all elements are absolute elements (and this can be considered as a symplectic polarity).

This is enough to get an induction started. Note that the problem only makes sense for finite dimensional projective spaces as infinite ones are never self dual.

We first prove some lemmas which are independent of the induction hypothesis.

Lemma 3.2. Let $\theta$ be a duality of a projective space of dimension $d>1$ with the property that every point is absolute. Then $\theta$ is a symplectic polarity.

Proof. Suppose by way of contradiction that $\theta$ is not a polarity. Then there is some point $x$ for which $x^{\prime}=x^{\theta^{2}} \neq x$. Consequently also $H:=x^{\theta}$ and $H^{\prime}={x^{\prime}}^{\theta}$ are different, and we can choose a point $y \in H, y \notin H^{\prime}$. Since lines are thick, there is a $z \in y x^{\prime}, z \notin\left\{x^{\prime}, y\right\}$. Since $z \in H$, we have $z^{\theta} \ni H^{\theta}=x^{\prime}$. Similarly, $x^{\prime} \in y^{\theta}$. By assumption $z \in z^{\theta}$ and $y \in y^{\theta}$. Hence the line $y z$ is in $z^{\theta} \cap y^{\theta}=(z y)^{\theta} \subseteq x^{\prime}$. This contradicts $y z \nsubseteq H^{\prime}$. Consequently $\theta$ is a polarity and hence a symplectic polarity as every point is an absolute one.

Lemma 3.3. If a line contains at least one non-absolute point, and $|\mathbb{K}|>2$, then it contains at least two non-absolute points.

Proof. Assume by way of contradiction that the line $L$ contains exactly one nonabsolute point $x$. Then $x^{\theta}$ intersects $L$ in some point $y \neq x$. Since, by assumption, $y \in y^{\theta}$, we see that $L^{\theta} \cap L=\{y\}$. If $u_{i} \in L, i=1,2, x \neq u_{i} \neq y$, then $u_{i}^{\theta}=\left\langle u_{i}, L^{\theta}\right\rangle=\left\langle L, L^{\theta}\right\rangle$, implying $u_{1}=u_{2}$, and so $|\mathbb{K}|=2$.

Proof of Theorem 3.1. In view of the induction procedure, we assume that for given $d>0$ the only domestic dualities of a projective space of dimension $d^{\prime}<d$ are the symplectic polarities for odd $d^{\prime}$, and we assume that $\theta$ is a domestic duality of a $d$-dimensional projective space $\Pi$.

In view of Lemma 3.2, it suffices to show that every point of $\Pi$ is absolute. Let, by way of contradiction, $x$ be a point which is not absolute, and let $H$ be its image under $\theta$. For any subspace $S$ in $H$, the image $\langle S, x\rangle^{\theta}=: S^{\prime}$ is a subspace 




\section{4. $J$-domestic collineations}

Now we consider collineations of projective spaces. Let us fix the projective space PG $(n, \mathbb{K})$, with $\mathbb{K}$ any skew field. Let $J$ be a subset of the type set. Define $J$ to be symmetric if, whenever $i \in J$, then $n-i-1 \in J$. Then clearly, if $J$ is not symmetric, then every collineation is $J$-domestic. Indeed, no flag of type $J$ is in that case opposite any flag of type $J$. Hence, from now on, we assume that $J$ is symmetric. We first prove two reduction lemmas. The first one reduces the question to type subsets of size 2, the second one reduces the question to single subspaces instead of pairs.

Lemma 4.1. Let $J$ be a symmetric subset of types for $\mathrm{PG}(n, \mathbb{K})$. Let $i$ be the largest element of $J$ satisfying $2 i<n$. Then a collineation $\theta$ of $\mathrm{PG}(n, \mathbb{K})$ is $J$-domestic if and only if it is $\{i, n-i-1\}$-domestic.

Proof. Clearly, if $\theta$ is $\{i, n-i-1\}$-domestic, then it is $J$-domestic. So assume that $\theta$ is $J$-domestic. Let $i$ be as in the statement of the lemma. Suppose that $\theta$ is not $\{i, n-i-1\}$-domestic and let $U$ and $V$ be subspaces of dimension $i$, $n-i-1$, respectively, such that $U \subseteq V$ with $\{U, V\}$ opposite $\left\{U^{\theta}, V^{\theta}\right\}$, i.e., $U \cap V^{\theta}=V \cap U^{\theta}=\emptyset$.

Now choose in $U$ any flag $\mathfrak{F}_{<i}$ of type $J_{<i}$, where with obvious notation, $J_{<i}=\{j \in J: j<i\}$. Let $\mathfrak{F}$ be an arbitrary extension of type $J$ of the flag $\mathfrak{F}_{<i} \cup\{U, V\}$. Then $\mathfrak{F}$ is opposite $\mathfrak{F}^{\theta}$ if and only if each subspace $W \in \mathfrak{F}$ of type $j>n-i-1$ is disjoint from the unique subspace $W^{\prime}$ of $\mathfrak{F}_{<i}^{\theta}$ of type $n-j-1$ and each subspace $Z \in \mathfrak{F}_{<i}$ of type $j<i$ is disjoint from the unique subspace $Z^{\prime}$ of $\mathfrak{F}^{\theta}$ of type $n-j-1$. The latter is equivalent with saying that each subspace $Y$ of $\mathfrak{F}$ of type $n-j-1>n-i-1$ is disjoint from the unique subspace $Y^{\prime} \in \mathfrak{F}_{<i}^{\theta^{-1}}$ of type $j$. So, we deduce that $\mathfrak{F}$ is opposite $\mathfrak{F}^{\theta}$ if, and only if, the flag $\mathfrak{F}_{>n-i-1}$ (with obvious notation) is opposite the two flags $\mathfrak{F}_{<i}^{\theta}$ and $\mathfrak{F}_{<i}^{\theta^{-1}}$. But one can always choose a flag opposite two given flags of the same type in any projective space. Indeed, this follows easily from the fact that we can always choose a subspace complementary to two given subspaces of the same dimension. Hence we have proved that $\theta$ is not $J$-domestic, a contradiction.

The lemma is proved.

So we have reduced the situation to symmetric type sets of two elements. With a similar technique, we reduce this further. But first a definition. For $i \leq n-i-1$ we say that a collineation is $i$ - $*$-domestic, if $\theta$ maps no subspace of dimension $i$ to a disjoint subspace.

Then we have: 


